\title{
REVISTAMATĒRIA
}

ISSN 1517-7076 artigos e-12886, 2020

\section{Avaliação do efeito da fadiga no módulo de elasticidade na flexão de painéis de madeira compensada}

\author{
Evaluation of the effect of fatigue on the \\ modulus of elasticity in static bending \\ of plywood panels
}

\author{
André Luiz Nonato Ferraz ${ }^{1}$, Giovana Gobatto Balanco ${ }^{2}$, \\ Vinícius Borges de Moura Aquino ${ }^{3}$, João Paulo Boff Almeida ${ }^{4}$, \\ André Luis Christoforo ${ }^{4}$, Francisco Antônio Rocco Lahr ${ }^{5}$
}

\footnotetext{
${ }^{1}$ Universidade do Estado do Mato Grosso, Departamento de Engenharia Civil, CEP: 78555-000, Sinop, MT, Brasil.

${ }^{2}$ Universidade de São Paulo, Escola de Engenharia de São Carlos, Departamento de Engenharia de Materiais, CEP: 13563-120, São Carlos, SP, Brasil.

${ }^{3}$ Universidade Federal do Sul e Sudeste do Pará, Instituto de Engenharia do Araguaia, CEP: 68560-000, Santana do Araguaia, PA, Brasil.

${ }^{4}$ Universidade Federal de São Carlos, Departamento de Engenharia Civil, CEP: 13565-905, São Carlos, SP, Brasil.

${ }^{5}$ Universidade de São Paulo, Escola de Engenharia de São Carlos, Departamento de Engenharia de Estruturas, CEP: 13566-590, São Carlos, SP, Brasil.

e-mail: andre.ferraz@unemat.br, giovanaagobatto@hotmail.com, aquino.vini@ hotmail.com, boff.joaopaulo@gmail.com; christoforoal@yahoo.com.br, frocco@sc.usp.br
}

\section{RESUMO}

Os painéis têm sido cada vez mais utilizados nas construções civis, rurais, entre outras, com destaque para os compensados pelo bom desempenho mecânico. Em muitas aplicações, os painéis podem estar sujeitos à ação de carregamentos cíclicos, e dessa forma, o conhecimento do efeito da fadiga é de fundamental importância. Todavia, as normas ABNT NBR ISO 2426 [12], NBR ISO 1096 [13], NBR 9489 [14] e NBR 9533 [15] que apresentam as classificações e os métodos de ensaios de painéis de madeira, não prescrevem requisitos para considerar o efeito da fadiga nas propriedades do material, o que motiva o desenvolvimento de pesquisas nessa temática. Este trabalho objetivou avaliar o efeito do número de ciclos de fadiga [NC] $(0$ - referência, $3600,7200,22500,45000,67500,90000)$ para a frequência de oscilação igual a 1,0 Hz nos valores do módulo de elasticidade na flexão (MOE) de painéis comerciais de madeira compensada de eucalipto. Foram fabricados seis corpos de prova para cada número de ciclos, resultando em 36 amostras. Os resultados evidenciaram reduções significativas do MOE a partir de 22500 ciclos, implicando que 0,3600 e 7200 ciclos forneceram resultados estatisticamente equivalentes. $\mathrm{O}$ modelo de regressão logarítmico mostrou ser o de melhor representatividade $\left(\mathrm{R}^{2}=64,28 \%\right)$, apresentando-se como alternativa na previsão do decaimento do MOE para ciclos de fadiga superiores aos avaliados nessa pesquisa.

Palavras-chave: Fadiga; Painel de madeira compensada; Análise de variância; Modelos de regressão.

\section{ABSTRACT}

The panels have been increasingly used in civil and rural buildings, among others, especially those plywood panels by good mechanical performance. In many applications, panels may be subject to cyclic loading, and thus, the knowledge of the effect of fatigue is of fundamental importance. However, the standards ABNT NBR ISO 2426 [12], NBR ISO 1096 [13], NBR 9489 [14] and NBR 9533 [15] which have the classifications and test methods for panels, do not consider the effect of fatigue in material properties, which motivates the development of researches in this area. The objective of this work was to evaluate the effect of the number cyclic fatigue load (NC) (0 - reference, 3600, 7200, 22500, 45000, 67500, 90000) for the oscillation frequency equal to $1.0 \mathrm{~Hz}$ in the bending modulus of elasticity (MOE) of commercial eucalyptus plywood panels. 
Six specimens were fabricated for each number of cycles, resulting in 36 samples. The results evidenced significant reductions of the MOE from 22500 cycles, implying that 0,3600 and 7200 cycles provided statistically equivalent results. The logarithmic regression model was the most representative $\left(R^{2}=64.28 \%\right)$, presenting as an alternative in the prediction of the MOE decay for fatigue cycles higher than those evaluated in this study.

Keywords: Fatigue; Plywood panel; Analysis of variance; Regression models.

\section{INTRODUÇÃO}

O uso de produtos engenheirados à base de madeira, como painéis MDF (Medium Density Fiberboard), LVL (Laminated Veneer Lumber), OSB (Oriented Strand Board) e painéis compensados de madeira consistem em alternativas no uso da madeira maciça em estruturas e construções rurais, podendo também ser aplicados na confecção de móveis, fôrmas para concreto armado, embalagens, entre outras [1- 7].

Dentre estes, pode-se destacar o uso do painel de madeira compensada, definido como painel de madeira composto por lâminas de madeiras sobrepostas em direções perpendiculares entre si, unidas através de adesivo, pressão e temperatura, com aplicações na construção civil, rural entre outras [8- 10].

O uso da madeira em estruturas no Brasil é regido pela norma brasileira ABNT NBR 7190 [11]. Juntamente a esta, os painéis de madeira compensada são normatizados pelas normas ABNT NBR ISO 2426 [12], NBR ISO 1096 [13], NBR 9489 [14] e NBR 9533 [15].

Para estruturas submetidas a cargas dinâmicas ou cíclicas, como pontes, viadutos e silos, deve-se levar em conta o efeito de fadiga no material [16, 17, 18,19]. Em ambas as normas brasileiras apresentadas, não há requisitos normativos para considerar o efeito de fadiga nas propriedades mecânicas do material, o que motiva o desenvolvimento de pesquisas nessa temática.

Das pesquisas envolvendo a fadiga, poucos são os trabalhos sobre a madeira sólida ([20], [21], [22], [18] e [23]), sendo ainda menor a quantidade de pesquisas relacionadas a painéis a base de madeira ([24-28]), com ênfase nos painéis aglomerados, de lascas orientadas (OSB) e de fibras (MDF).

Com o objetivo de determinar valores máximos de tensão em que possam ser submetidos painéis aglomerados, compensados, MDF e OSB, BAO et al. [27] analisaram o comportamento à fadiga destes materiais por meio do ensaio de flexão estática. Cinco níveis de tensão, correspondentes a uma porcentagem do módulo de resistência (MOR), foram aplicados aos corpos de prova. Os autores concluíram que, de modo geral, o número de ciclos, suportados pelo material, diminuiu à medida que se aumentou o nível de tensão. Ademais, considerando o nível de tensão igual a $30 \%$, todos os materiais investigados suportaram ciclos de carregamento superiores a 1000000 .

THOMPSON et al. [25] e THOMPSON et al. [26] realizaram trabalhos para avaliação do desempenho de painéis OSB, aglomerados e MDF submetidos à fadiga em ensaios de flexão a quatro pontos e as mudanças nas propriedades dos mesmos durante os ciclos de carregamento. Segundo os autores, em baixos níveis de carga o OSB e o aglomerado apresentaram comportamento semelhante em relação à fadiga, e o OSB apresentou maior rigidez à flexão em relação aos demais painéis estudados, assim como esperado.

PRITCHARD et al. [24] estudaram o efeito de dois teores de umidade relativa (ambientes com $65 \mathrm{e}$ $85 \%$ de umidade) no módulo de elasticidade (MOE) e no módulo de resistência (MOR) em painéis OSB, aglomerado e MDF. Como esperado, o ambiente com teor de umidade relativa de $85 \%$ foi mais prejudicial ao OSB em relação ao ambiente com $65 \%$, diminuindo significativamente os valores das propriedades estudadas. Além disso, o MDF apresentou melhores resultados em termos de perdas das propriedades comparando os dois ambientes com umidades relativas diferentes. De acordo com os autores isso se deve às características dos painéis, já que o MDF é mais homogêneo que o OSB e aglomerado.

Por fim, DEL MENEZZI et al. [28] avaliaram o efeito do carregamento cíclico nas propriedades de flexão estática (MOE e MOR) de painéis comerciais de MDF e MDP (Medium Density particleboard). Para tanto, em todos os ciclos de carregamento adotados (20000, 40000 e 80000), os autores submeteram o material a um carregamento com intensidade correspondente a $25 \%$ do MOR e frequência de oscilação de $0,45 \mathrm{~Hz}$. Concluiu-se que os diferentes ciclos de carregamento não causaram efeito deletério no MOE e MOR para ambos os painéis analisados.

Visando enriquecer as normas brasileiras pertinentes ao uso de produtos à base de madeira engenheirada no tocante ao efeito de fadiga em painéis de madeira compensada, este trabalho objetivou avaliar o comportamento de painéis comerciais de madeira compensada na fadiga à flexão, considerando-se o efeito do número de ciclos $(0,3600,7200,22500,45000,67500,90000)$ para a frequência de oscilação igual a $1,0 \mathrm{~Hz}$ na determinação módulo de elasticidade (MOE). 


\section{MATERIAIS E MÉTODOS}

Para os ensaios de carregamento cíclico, foram utilizados corpos de prova $(50 \times 200 \times 8 \mathrm{~mm})$ extraídos de 6 painéis comerciais compensados de madeira de eucalipto (dimensões $2200 \times 1600 \mathrm{~mm}$ ) proveniente da empresa Ecoplac®, com espessura igual a $8 \mathrm{~mm}$ e fabricados com resinas MR-Reutilizável e fenólica WBP (weather and boil proof) $100 \%$ resistente à água, informações estas disponibilizadas pelo fabricante.

O módulo de elasticidade (MOE) (Equação 1) foi calculado conforme o disposto na ABNT NBR 7190 [11] e ABNT NBR 9533 [15], porém, o ensaio de flexão estática a três pontos foi adaptado para a condição não destrutiva, admitindo deslocamento máximo igual a L/200 (ABNT NBR 7190 [11]). Tal deslocamento assegura a linearidade física e geométrica do material, sendo comumente empregado na determinação do MOE em ensaios não destrutivos, como nos trabalhos de CHRISTOFORO et al. [29], ICIMOTO et al. [30], ZANGIÁCOMO et al. [31], SEGUNDINHO et al. [32], e LAHR et al. [33]. No ensaio de carregamento cíclico (fadiga), como o modelo esquemático (Figura 1b) dos apoios impossibilita a livre rotação do corpo de prova na seção dos apoios, uma vez que os parafusos pressionaram as amostras, o limite de deslocamento adotado (garantia de linearidade física e geométrica) foi igual a L/300. Cabe destacar que os parafusos não penetraram nos corpos de prova.

$$
M O E=\frac{F \cdot L^{3}}{4 \cdot \delta \cdot b \cdot h^{3}}
$$

Da Equação 1, F representa a força obtida no ensaio de flexão associada ao deslocamento relativo ( $\delta=\mathrm{L} / 200)$, L é o comprimento útil da amostra (distância entre apoios) e b (base) e h (altura) são dimensões da seção transversal das amostras.

De modo a expor os painéis a condições mais severas, a frequência de oscilação adotada na presente pesquisa foi igual a $1,0 \mathrm{~Hz}$, superior à frequência de $0,4 \mathrm{~Hz}$ e $0,45 \mathrm{~Hz}$ utilizada, respectivamente, por GUIMARÃES et al. [22] e DEL MENEZZI et al. [28]. Os ciclos de fadiga adotados neste trabalho foram: 0 (referência), 3600, 7200, 22500, 45000, 67500 e 90000, o que resultou em seis tratamentos experimentais distintos juntamente com a condição de referência (Tabela 1). O número máximo de ciclos desta pesquisa é inferior ao máximo (100000) utilizado por GUIMARÃES et al. [22], entretanto, cabe destacar que a pesquisa de Guimarães et al. [22] foi realizada considerando-se a madeira sólida.

Tabela 1: Tratamentos idealizados.

\begin{tabular}{c|c|c}
\hline TRATAMENTOS & FREQUÊNCIA (Fr) & NÚMERO DE CICLOS (NC) \\
\hline Ref & 0 & 0 \\
\hline 1 & $1,0 \mathrm{~Hz}$ & 3600 \\
\hline 2 & $1,0 \mathrm{~Hz}$ & 7200 \\
\hline 3 & $1,0 \mathrm{~Hz}$ & 22500 \\
\hline 4 & $1,0 \mathrm{~Hz}$ & 45000 \\
\hline 5 & $1,0 \mathrm{~Hz}$ & 67500 \\
\hline 6 & $1,0 \mathrm{~Hz}$ & 90000 \\
\hline
\end{tabular}

Foram utilizados seis corpos de prova para cada um dos seis níveis do número de ciclos, resultando em 36 amostras ao todo. Em todos os casos, o MOE foi inicialmente obtido (metodologia não destrutiva) antes da exposição das amostras aos ciclos de fadiga, resultando na condição de referência. Posteriormente a essa etapa, as amostras foram levadas para a máquina de fadiga (Figura 1a), e fadigadas as amostras (para cada número de ciclos), essas foram posteriormente ensaiadas na flexão estática à três pontos, com o auxílio da máquina de ensaios EMIC (capacidade de carga de $30 \mathrm{kN}$ ), para obtenção do módulo de elasticidade. 


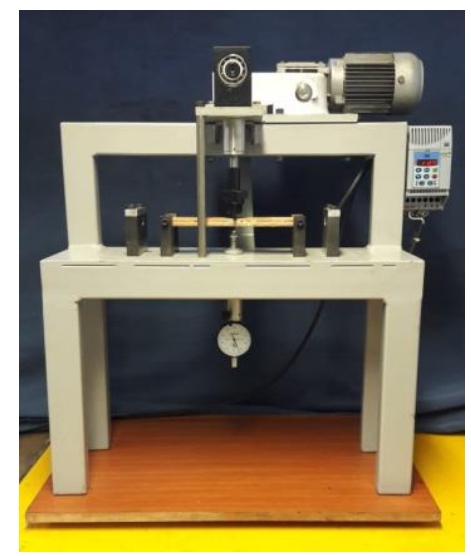

(a)

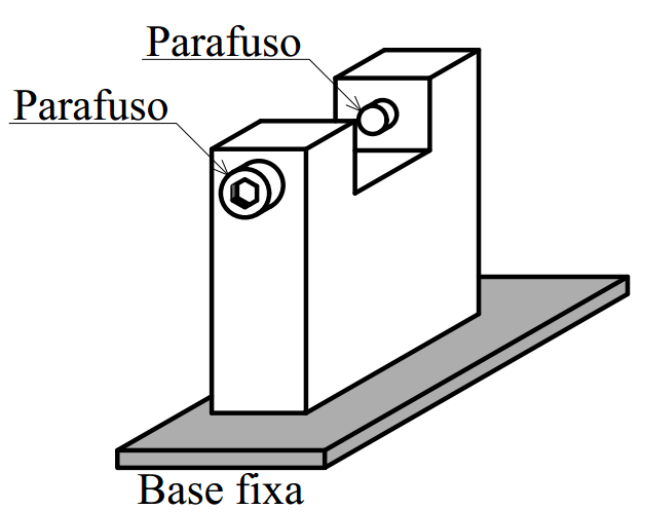

(b)

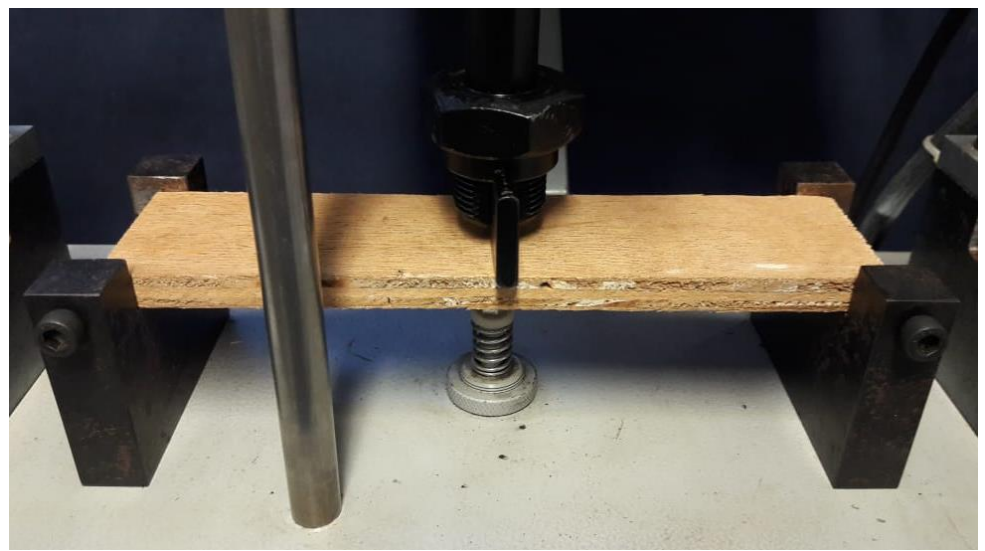

(c)

Figura 1: Máquina de fadiga (a) Modelo esquemático dos apoios (b) Corpo de prova (c).

Cabe destacar que as amostras dos painéis foram avaliadas tanto no ensaio de fadiga quanto no de flexão estática com as faces dispostas na mesma posição, eliminado assim as possíveis variabilidades decorrentes da anisotropia da madeira que poderiam interferir nos resultados do $\mathrm{MOE}[4,30]$.

A análise de variância (ANOVA), ao nível de 5\% de significância, foi utilizada para investigar a influência do número de ciclos de fadiga nos valores do módulo de elasticidade na flexão estática dos painéis compensados. Pelas hipóteses formuladas, P-valor (probabilidade P) maior ou igual ao nível de significância ( $\mathrm{P}$-valor $\geq 0,05)$ implica que os ciclos de fadiga não influenciam nos valores do MOE de forma significativa (médias estatisticamente equivalentes), sendo significativos em caso contrário ( $\mathrm{P}$-valor $<0,05)$.

Para validação da ANOVA foram utilizados os testes de normalidade de Anderson-Darling (AD) e o da homogeneidade de variâncias de Bartlett $(\mathrm{Bt})$ sobre os resíduos. Pelas formulações dos testes, P-valor maior ou igual ao nível de significância implica que os resíduos apresentam normalidade na distribuição e que as variâncias são homogêneas, validando o modelo da ANOVA.

Constatada a significância do fator NC nos valores do MOE, na sequência foi-se utilizado o teste de contraste de Tukey para o agrupamento dos níveis desse fator. Do teste de Tukey, também avaliado ao nível de 5\% de significância, A denota o nível do fator de maior valor médio, B o segundo maior valor médio e assim sucessivamente, e letras iguais implicam em níveis do fator com médias equivalentes entre si.

Avaliada a evolução dos valores do MOE em função do número de ciclos de fadiga pela ANOVA, modelos de regressão (Tabela 2), também fundamentados na ANOVA, foram utilizados na estimativa dos valores do MOE, sendo o melhor ajuste eleito em função do maior valor do coeficiente de determinação ajustado $\left(\mathrm{R}^{2}\right)$. 
Tabela 2: Modelos de regressão utilizados na estimativa do módulo de elasticidade na flexão estática (MOE) dos painéis em função do número de ciclos de fadiga (NC).

\begin{tabular}{c|c}
\hline TIPO DE AJUSTE & MODELO \\
\hline Polinomial Linear [Lin] & $M O E=a+b \cdot N C$ \\
\hline Exponencial [Exp] & $M O E=a \cdot e^{b \cdot N C}$ \\
\hline Logarítmica [Log] & $M O E=a+b \cdot \operatorname{Ln}(N C)$ \\
\hline Geométrica [Geo] & $M O E=a \cdot N C^{b}$ \\
\hline
\end{tabular}

A ANOVA dos modelos de regressão, também avaliada ao nível de 5\% de significância, possibilita avaliar se o modelo e se os coeficientes são significativos (P-valor $<0,05$ ) na estimativa dos valores do MOE.

\section{RESULTADOS E DISCUSSÕES}

A Figura 2 ilustra os valores médios e os intervalos de confiança da média (95\% de confiabilidade) dos módulos de elasticidade (MOE) na flexão estática dos painéis compensados em função de número de ciclos de fadiga, cabendo destacar que o coeficiente de variação (CV) do MOE dos painéis variou entre 6,28\% a $14,51 \%$.

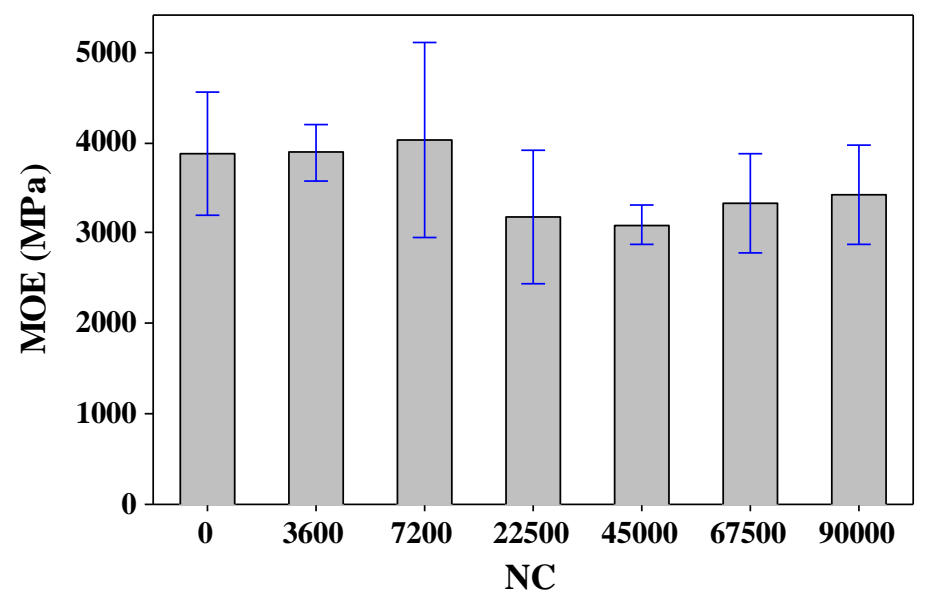

Figura 2: Resultados do módulo de elasticidade em função do número de ciclos de fadiga.

Vale comentar que não existem estudos versando sobre os efeitos da fadiga nas propriedades de flexão de painéis compensados, o que impossibilita a comparação dos resultados aqui obtidos.

As médias do MOE para 0, 3600 e 7200 ciclos foram muito próximas, com redução de aproximadamente $21 \%$ no valor do módulo de elasticidade de 7200 para 22500 ciclos. Entre 22500 a 90000 ciclos não foram notadas grandes diferenças nos valores dessa propriedade (Figura 2).

No ensaio de fadiga, o intervalo de carregamento foi de 49,92 $\mathrm{N}$ a 39,68 N, sendo que a razão de tensão, obtida pelo quociente entre os valores máximo e mínimo de tensão, correspondeu a 1,26.

Os P-valores do teste de normalidade $[\mathrm{AD}](0,582)$ e de homogeneidade de variâncias $[\mathrm{Bt}](0,530)$ foram ambos superiores ao nível de significância adotado $(0,05)$, validando assim o modelo da ANOVA. O Pvalor da ANOVA foi igual a 0,02 $(<0,05)$, evidenciando que o número de ciclos estipulado afetou de forma significativa no valor do MOE dos painéis compensados. A Tabela 3 apresenta os resultados do teste de Tukey. 
Tabela 3: Resultados do teste de Tukey para o MOE dos painéis compensados em função dos ciclos de fadiga.

\begin{tabular}{c|c|c|c|c|c|c|c}
\hline NC & 0 & 3600 & 7200 & 22500 & 45000 & 67500 & 90000 \\
\hline Grupos & A & A & A & B & B & B & B \\
\hline
\end{tabular}

Da Tabela 3, nota-se que os três primeiros níveis do fator NC $(0,3600,7200$ ciclos) resultaram em médias do MOE estatisticamente equivalentes entre si, consistindo nos maiores valores médios dessa propriedade. O MOE sofreu redução de $21 \%$ a partir de 22500 ciclos de fadiga, mas permanecendo inalterado entre 22500 a 90000 ciclos (médias equivalentes). Tal redução é uma informação importante a ser considerada em futuras revisões dos documentos normativos brasileiros no que diz respeito a projetos de painéis compensados sujeitos à ação de cargas cíclicas. A redução do MOE pode ser justificada pelo fato da madeira apresentar alterações em sua organização celular quando submetida a carregamentos cíclicos ou contínuos [34].

A Tabela 4 apresenta os resultados dos modelos de regressão para a estimativa do MOE em função do número de ciclos.

Tabela 4: Modelos de regressão para a estimativa do MOE.

\begin{tabular}{c|c|c|c|c}
\hline \multirow{2}{*}{ MODELOS } & \multicolumn{2}{|c|}{ PARÂMETROS } & \multirow{2}{*}{$\begin{array}{c}\text { P-VALOR } \\
\text { (ANOVA) }\end{array}$} & \multirow{2}{*}{$\mathbf{R}^{\mathbf{2}}$} \\
\cline { 2 - 3 } & $\mathbf{a}$ & $\mathbf{b}$ & 0,264 & $29,66 \%$ \\
\hline$M O E=a+b \cdot N C$ [Linear] & 3729,92 & $-0,0060$ & 0,285 & $27,51 \%$ \\
\hline$M O E=a \cdot e^{b \cdot N C}[$ Exponencial] & 3706,28 & 0,000 & 0,036 & $64,28 \%$ \\
\hline$M O E=a+b \cdot \operatorname{Ln}(N C)[$ Logarítmico] & 5785,12 & $-227,99$ & 0,043 & $56,41 \%$ \\
\hline$M O E=a \cdot N C^{b}[$ Geométrico] & 6554,08 & $-0,0631$ & \\
\hline
\end{tabular}

Da Tabela 4, os modelos linear e exponencial não foram considerados significativos na estimativa do MOE pela ANOVA (P-valor $\geq 0,05$ ). Os modelos logarítmico e geométrico foram considerados significativos $(\mathrm{P}$-valor $<0,05)$, sendo o melhor ajuste (Figura 3) obtido com o uso do modelo logarítmico $\left(\mathrm{R}^{2}=64,28 \%\right)$.

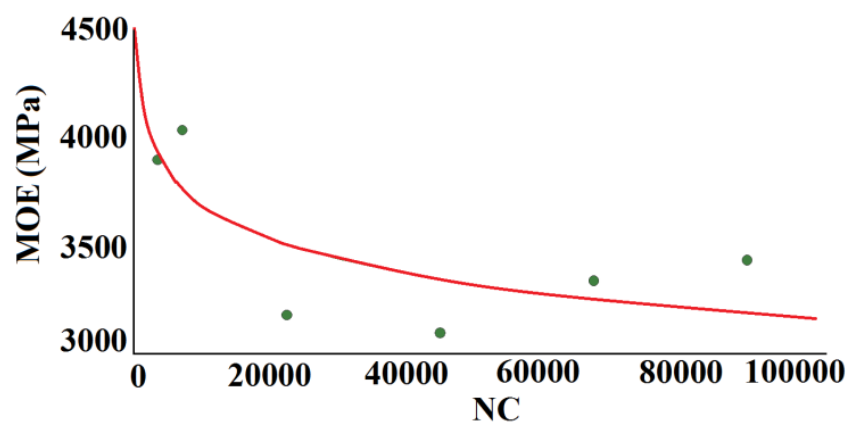

Figura 3: Melhor ajuste testado - modelo logarítmico.

Pelo modelo de regressão logarítmico (Tabela 4), considerando-se o dobro da frequência utilizada nessa pesquisa (200000), o valor estimado do MOE é igual a 3003,36 MPa, correspondendo a 85,80\% do valor estimado $(3501,27 \mathrm{MPa})$ do MOE para 22500 ciclos. Para modelos mais precisos na estimativa do MOE faz-se necessário utilizar um número maior de amostras, de ciclos (superiores a 100000) e de frequências (superior a $1 \mathrm{~Hz}$ ), que podem promover reduções mais ou menos acentuadas nessa propriedade, e que deve ser o foco de pesquisas futuras.

\section{CONCLUSÕES}

Os resultados da presente pesquisa permitem concluir que houve redução significativa dos valores médios do módulo de elasticidade em função dos ciclos de fadiga avaliados.

As peças ensaiadas com 0 (referência), 3600 e 7200 ciclos apresentaram módulo de elasticidade estatisticamente equivalentes, havendo uma redução considerável de $21 \%$ no MOE a partir de 22500 ciclos, entretanto, de 22500 a 90000, as médias dos valores do MOE mantiveram-se equivalentes. 
A redução de $21 \%$ nos valores do MOE a partir de 22500 ciclos é uma informação importante para fundamentar futuras revisões das normativas brasileiras no que se refere a elaboração de projetos em que os painéis compensados são expostos a carregamentos cíclicos. No entanto, se fazem necessários outros estudos que considerem mais categorias de painéis compensados comerciais, maiores números de ciclos e de amostras ensaiadas por ciclo, análise da influência de parâmetros como umidade, efeito de escala e outros que naturalmente ocorrem nas estruturas, a fim de estabelecer modelos mais abrangentes que conduzam a estimativas mais precisas do modulo de elasticidade.

\section{AGRADECIMENTOS}

Os autores agradecem a Coordenação de Aperfeiçoamento de Pessoal de Nível Superior e ao Conselho Nacional de Desenvolvimento Científico e Tecnológico $(\mathrm{CNPq})$ pelo apoio no desenvolvimento das pesquisas realizadas.

\section{BIBLIOGRAFIA}

[1] PALMA, H.A.L., BALLARIN, A.W. "Propriedades físicas e mecânicas de paineis LVL de Eucalyptus grandis". Ciência Florestal, v. 21, n. 3, pp. 559-566, 2011.

[2] FERRO, F.S., ALMEIDA, T.H., ALMEIDA, D.H., et al., "Physical Properties of OSB Panels Manufactured with CCA and CCB Treated Schizolobium amazonicum and Bonded with Castor Oil Based Polyurethane Resin”. International Journal of Materials Engineering, v. 6, n. 5, pp. 151-154, 2016.

[3] IWAKIRI, S., TRIANOSKI, R., FONTE, A.P.N., et al., "Potencial de uso de madeiras de Dinizia excelsa Ducke e Protium puncticulatum J. F. Macbr para produção de painéis EGP”. Scientia Forestalis, v. 44, n. 111, pp. 709-717, 2016.

[4] FERREIRA, B.S., SILVA, J.V.F., CAMPOS, C.I. "Static bending strength of heat-treated and chromated copper arsenate-treated plywood”. BioResources, v. 12, n. 3, pp. 6276-6282, 2017.

[5] FREITAS, J.F., SOUZA, A.M., GRANCO, L.A.M.N., et al., "A Preliminary Study about the Utilization of Cajueiro and Amescla for MDF Panels Production". International Journal of Materials Engineering, v. 7, n. 2, pp. 21-24, 2017.

[6] SOUZA, A.M., NASCIMENTO, M.F., ALMEIDA, D.H., et al., "Wood-based composite made of wood waste and epoxy based ink-waste as adhesive: a cleaner production alternative". Journal of Cleaner Production, v. 193, pp. 549-562, 2018.

[7] SUSAINATHAN, J., EYMA, F., LUYCKER, E., et al., "Experimental investigation of impact behavior of wood-based sandwich structures". Composites Part A: Applied Science and Manufacturing, v. 109, pp. 1019, 2018.

[8] FERREIRA, B.S., SILVEIRA, M., CAMPOS, I. “Análise do desempenho físico-mecânico de compensados produzidos com adesivos a base de PVA". Madeira: Arquitetura e Engenharia, v. 12, n. 19, pp. 13-22, 2011.

[9] ZHOU, X., PIZZI, A. "Pine tannin based adhesive mixes for plywood". International Wood Products Journal, v. 5, n. 1, pp. 27-32, 2014.

[10] DEMIRKIR, C., COLAKOGLU, G., COLAK, S., AYDIN, I., CANDAN, Z. "Influence of aging procedure on bonding strength and thermal conductivity of plywood panels". Acta Physica Polonica A, v. 129, n. 6, pp. 1230-1234, 2016.

[11] ASSOCIAÇÃO BRASILEIRA DE NORMAS TÉCNICAS (ABNT). NBR 7190: "Projeto de estruturas de madeira". Rio de Janeiro, 1997.

[12] ASSOCIAÇÃO BRASILEIRA DE NORMAS TÉCNICAS (ABNT). NBR ISO 2426: "Madeira compensada - Classificação pela aparência superficial”. Rio de Janeiro, 2006.

[13] ASSOCIAÇÃO BRASILEIRA DE NORMAS TÉCNICAS (ABNT). NBR ISO 1096: "Madeira compensada - Classificação". Rio de Janeiro, 2006.

[14] ASSOCIAÇÃO BRASILEIRA DE NORMAS TÉCNICAS (ABNT). NBR 9489: “Condicionamento de corpos de prova de compensados para ensaios - Requisitos". Rio de Janeiro, 2011.

[15] ASSOCIAÇÃO BRASILEIRA DE NORMAS TÉCNICAS (ABNT). NBR 9533: "Compensado - Determinação da resistência à flexão estática". Rio de Janeiro, 2012. 
[16] SALMI, A., MONTONEN, R., SALMINEN, L.I., SUURONEN, J.P., SERIMAA, R., HÆGGSTRÖM, E. "Cyclic impulsive compression loading along the radial and tangential wood directions causes localized fatigue". Journal of Applied Physics, v. 112, n. 12, 2012.

[17] SASAKI, Y., OYA, A., YAMASAKI, M. "Energetic investigation of the fatigue of wood". Holzforschung, v. 68, n. 7, pp. 843-848, 2014.

[18] LI, Y., LAM, F. "Low cycle fatigue tests and damage accumulation models on the rolling shear strength of cross-laminated timber". Journal of Wood Science, v. 62, n. 3, pp. 251-262, 2016.

[19] NAGASE, K., KOBAYASHI, K., YASUMURA, M. "Estimation of failure lifetime in plywood-totimber joints with nails and screws under cyclic loading". Journal of Wood Science, v. 0, n. 0, pp. 1-13, 2018.

[20] MACÊDO, A.N., CALIL JUNIOR, C. "Fadiga em emendas dentadas em madeira laminada colada". Cadernos de Engenharia de Estruturas, v. 9, n. 37, pp. 95-126, 2007.

[21] MIYAZAKI, J., NAKANO, T. "Fracture behavior of laminated wood bonded with water based polymer-isocyanate resin and resorcinol-formaldehyde resin under impact fatigue". Journal of Applied Polymer Science, v. 109, pp. 276-281, 2008.

[22] GUIMARÃES, L.E.S., BUSSINGUER, A.P., DEL MENEZZI, C.H.S., et al.,"Efeito do carregamento cíclico sobre as propriedades de flexão e ultrassom em madeiras de quatro espécies florestais". Scientia Forestalis, v. 40, n. 96, pp. 473-481, 2012.

[23] MYSLICKI, S., VALLÉ, T., WALTHER, F. "Short-time procedure for fatigue assessment of beech wood and adhesively bonded beech wood joints". Materials and Structures, v. 49, n. 6, pp. 2161-2170, 2016.

[24] PRITCHARD, J., ANSELL, M.P., THOMPSON, R.J.H., BONFIELD, P.W. "Effect of two relative humidities on the MOE, MOR and fatigue life of MDF, OSB and chipboard". Wood Science and Technology, v. 35, pp. 395-424, 2001.

[25] THOMPSON, R.J.H., ANSELL, M.P., BONFIELD, P.W., et al., "Fatigue in wood-based panels. Part 1: The strength variability and fatigue performance of OSB, chipboard and MDF". Wood Science and Technology, v. 36, pp. 255-268, 2002.

[26] THOMPSON, R.J.H., ANSELL, M.P., BONFIELD, P.W., et al., "Fatigue in wood-based panels. Part 2: property changes during fatigue cycling of OSB, chipboard and MDF". Wood Science and Technology, v. 39, pp. 311-325, 2005.

[27] BAO, Z.; ECKELMAN, C. "Fatigue life and design stresses for wood composites used in furniture". Forest Products Journal, v. 45, n. 7/8, p. 59-63, 1995.

[28] DEL MENEZZI, C.H.S., SANTOS, C.M.T., FERRAZ, J.M., et al., "Efeito do carregamento cíclico sobre as propriedades de flexão de painéis comerciais de MDF e MDO". Cerne, v. 17, n.3, pp. 403-409, 2011.

[29] CHRISTOFORO, A.L., PANZERA, T.H., ARAUJO, V.A., et al., "Timber beam repair based on polymer-cementitious blends". Engenharia Agrícola, v. 37, n. 2, pp. 366-375, 2017.

[30] ICIMOTO, F. H., FERRO, F.S., ALMEIDA, D.H., et al., "Influence of specimen orientation on determination of elasticity in static bending". Maderas. Ciencia y tecnología, v. 17, n.5, pp. 229-238, 2015.

[31] ZANGIÁCOMO, A.L., CHRISTOFORO, A.L., LAHR, F.A.R. "Módulo de elasticidade aparente em vigas roliças estruturais de madeira Pinus elliotti”. Ambiente Construído, v. 14, n.1, pp. 7-13, 2014.

[32] SEGUNDINHO, P.G.A., ZANGIÁCOMO, A.L., CARREIRA, M.R., et al., "Avaliação de vigas de madeira laminada colada de cedrinho (Erisma uncinatum Warm.)". Cerne, v. 19, n.3, pp. 441-449, 2013.

[33] LAHR, F.A.R., CHRISTOFORO, A.L., VARANDA, L.D., et al., "Shear and longitudinal modulus of elasticity in wood: relations based on static beding test". Acta Scientiarum, v. 39, n.4, pp. 433-437, 2017.

[34] FRIHART, C.R. "Wood adhesion and adhesives". In: ROWELL, R. Wood chemistry and wood composites. New York: CRC, 2005.

\section{ORCID}

André Luiz Nonato Ferraz

Giovana Gobatto Balanco

Vinícius Borges de Moura Aquino

João Paulo Boff Almeida

André Luis Christoforo

Francisco Antônio Rocco Lahr https://orcid.org/0000-0001-7942-8883

https://orcid.org/0000-0002-2544-3743

https://orcid.org/0000-0003-3483-7506

https://orcid.org/0000-0001-8667-1718

https://orcid.org/0000-0002-4066-080X

https://orcid.org/0000-0002-3510-8498 